\title{
Conservation and Diversification of Floral Homeotic MADS-box Genes in
}

\section{Eustoma grandiflorum}

\author{
Motoyuki Ishimori and Saneyuki Kawabata* \\ Graduate School of Agricultural and Life Sciences, The University of Tokyo, Bunkyo-ku, Tokyo 113-8657, Japan
}

The MADS-box gene family is one of the largest transcription factor gene families in plants and is necessary at

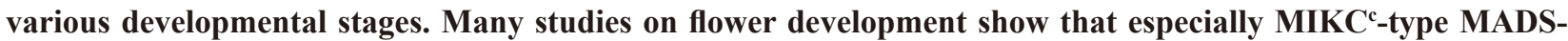
box genes are essential for proper floral organ development. We identified and characterized MIKC ${ }^{c}$-type MADS-box genes expressed in Eustoma grandiflorum flowers. Twenty-three genes were identified and grouped into 10 clades, which were characterized by conserved specific motifs. Phylogenetic analysis indicated the diversification of $A G / P L E, A P 3 / D E F, P I / G L O$, and $S E P$ clades and the occurrence of recent gene duplication events. The floral organ-specific expression patterns were partly diversified within the gene members of $A P 3 / D E F$ and $S E P$ clades, while they were conserved in $A G / P L E$ and $P I / G L O$ clades. These results suggest that genes with conserved expression as well as those with diversified expression contribute to specifying floral organ identity in E. grandiflorum.

Key Words: ABC model, floral organ identity, gene duplication, lisianthus, transcription factor family.

\section{Introduction}

For several decades, many plant scientists have tried to elucidate the molecular mechanisms of flower development. One of the most fruitful results from this research was the discovery of MADS-box transcription factor family genes that play important roles in floral organ development (Sommer et al., 1990; Yanofsky et al., 1990). This finding led to the floral "ABC" model, which has been modified as the "ABCE" or "ABCDE" model (Coen and Meyerowitz, 1991; Krizek and Fletcher, 2005). Most of the gene members of the "ABCDE" model belong to the MADS-box family, except for the APETALA2 (AP2) gene (Goto and Meyerowitz, 1994; Jack et al., 1992; Jofuku et al., 1994; Mandel et al., 1992; Pelaz et al., 2000; Pinyopich et al., 2003; Yanofsky et al., 1990). This model explains the determination of floral organ identity by combinatory functions of MADS-box proteins classified into different classes (A, B, C, D, and E). The combinations of $\mathrm{A}+\mathrm{E}, \mathrm{A}+\mathrm{B}+\mathrm{E}, \mathrm{B}+\mathrm{C}+\mathrm{E}, \mathrm{C}+\mathrm{E}$, and $\mathrm{D}+\mathrm{E}$ specify sepal, petal, stamen, carpel, and ovule identity, respectively (Immink et al., 2010). The A class gene includes APETALA1 (AP1), B class includes APETALA3 (AP3)

Received; October 22, 2013. Accepted; January 7, 2014.

First Published Online in J-STAGE on March 12, 2014.

* Corresponding author (E-mail: ayuki@mail.ecc.u-tokyo.ac.jp). and PISTILLATA (PI), C class includes AGAMOUS $(A G)$, D class includes SEEDSTICK $(S T K)$, and E class includes SEPALLATA1-4 (SEP) in Arabidopsis (Krizek and Fletcher, 2005).

The MADS-box genes comprise a large gene family in plants. For example, 107 MADS-box genes were identified in Arabidopsis (Pařenicová et al., 2003). Both plant and animal MADS-box genes can be classified into type I and type II (Alvarez-Buylla et al., 2000). Plant type II MADS-box genes belong to "MIKC-type", named after their four specific domains, a MADS-box (M), intervening (I), keratin-like (K), and C-terminal (C) domains (Kaufmann et al., 2005). Of the four domains, the MADS-box domain is especially important for DNA binding and dimerization, and is highly conserved throughout eukaryotes. MIKC-type MADS-box genes are further divided into MIKC $\mathrm{MIype}^{\mathrm{c}}$ and $\mathrm{MIKC}^{*}$ type genes, but many seed plant type II and all $\mathrm{ABCDE}$ MADS-box genes belong to the former group.

Eustoma grandiflorum is one of the most popular species in the cut flower market and many cultivars with different flower shapes and colors, and different growth characteristics have been developed. In spite of its economic value, genomic information about this species is still limited. We previously analyzed the transcriptome of E. grandiflorum during flower development (Kawabata et al., 2012). The transcriptome was expected to include a number of genes involved in floral organ development, 
such as the ABCDE MADS-box genes. To examine the involvement of MADS-box genes in flower development in E. grandiflorum, we identified MIKC ${ }^{c}$-type MADSbox genes from the above transcriptome and characterized their floral organ-specific expressions. MIKC ${ }^{c}$-type MADS-box genes included not only ABCDE genes but also other genes involved in the regulation of flowering time.

\section{Materials and Methods}

\section{Plant materials}

E. grandiflorum 'Azuma no Murasaki' (Sakata Seed, Kanagawa, Japan) was grown in a growth chamber controlled at $20-22^{\circ} \mathrm{C}$ with a $16 \mathrm{~h}$ photoperiod. After two months, they were transplanted to pots in March 2013 and grown in a greenhouse under natural light conditions until flowering. Flowers at the $12 \mathrm{~mm}$ bud stage were collected in June and dissected into sepals, petals, stamens, and carpels. The samples were frozen in liquid nitrogen immediately and kept at $-60^{\circ} \mathrm{C}$ until RNA extraction.

\section{Gene identification}

Previously sequenced reads of the floral transcriptome (Kawabata et al., 2012) were re-assembled using a GS De Novo Assembler (Roche Applied Science, Mannheim, Germany). Contigs containing the MIKC ${ }^{c}$-type MADS domain were screened by the hidden Markov model (HMM) approach using the HMM-FRAME program (Zhang and Sun, 2011). The HMM profile of the MADS domain was constructed with the HMMER program (Eddy, 2009), using the multiple alignment file of the conserved MADS domain (Entry name: MADS_BOX_1, accession number; PS00350) reposited at PROSITE (http://prosite.expasy.org/). The identified MADS-box genes were aligned by Clustal X version 2.0 (Larkin et al., 2007), and the redundant sequences were removed manually. The original sequence reads that corresponded to each identified MADS-box sequence were collected by conducting a blast search. These reads were mapped to the contigs to manually correct sequence errors and extend contig length. The ORFs were predicted using FrameDP (Gouzy et al., 2009) with a complete set of Arabidopsis proteins (TAIR_10_pep_20101214 updated, ftp://ftp.arabidopsis.org/home/tair/Sequences/ blast_datasets/TAIR10_blastsets/) as the reference database.

\section{Phylogenetic analysis}

Phylogenetic trees for the identified MADS-box genes were constructed along with related genes of other species retrieved from the GenBank database using MEGA v5.2 (Tamura et al., 2011). Clustal W (Larkin et al., 2007) was used for aligning the full-length amino acid sequences.

\section{Motif prediction}

MEME (Bailey et al., 2006) was used for detecting motifs in E. grandiflorum MADS-box proteins. InterProscan (Quevillon et al., 2005) was employed to examine whether the predicted motifs were known domains. Schematic diagrams of protein domain structures were drawn using DOG 2.0 (Ren et al., 2009).

\section{Expression analysis}

Total RNA was extracted from the tissue using a Spectrum Plant Total RNA Kit (Sigma-Aldrich, St. Louis, MO, USA). The cDNA was reverse-transcribed

Table 1. Primers used in this study.

\begin{tabular}{|c|c|c|}
\hline Gene & Forward primer sequence $\left(5^{\prime}-3^{\prime}\right)$ & Reverse primer sequence $\left(5^{\prime}-3^{\prime}\right)$ \\
\hline EgPLE1 & tactgataccaccagcacaggat & tttcctcagcttgtctgattctt \\
\hline$E g P L E 2$ & gtgagtactgataccaccagtatagg & cttatatgcttccccagcttttt \\
\hline EgPLE3 & gcttctgctgataccacyaattcgg & ctttccttagcttgctggettgttg \\
\hline EgSTK & gaccgttacaggaaagcaacttcag & gcttaaaccetctcccatcagatgc \\
\hline EgAPla & gaagacaatggcgcaacaac & gaggaaaggtgtcgaatttgc \\
\hline$E g A P 1 b$ & tgtccettcacttgttctctca & ctacaggttggtatgcgtcact \\
\hline$E g D E F 1$ & ggctctgatcttaccacctttg & taaagagatgagggagacaagacc \\
\hline$E g D E F 2$ & cctaaccegeccagttttat & agcaatgcgaaggtggtaag \\
\hline$E g D E F 3$ & agaaccgaaccaacagacaagt & ttgaacagcccatttcttcttt \\
\hline EgTM6 & gtactctttcgecttgcagtct & taatctccaagcctgaggttct \\
\hline$E g G L O 1$ & gaaaatgctagaggatcagcacaagcag & tcgaaatgcaaaaggcatatgggaatc \\
\hline$E g G L O 2$ & gaatgctagaggaggagcacaagcac & gaaatgcaaaaggcatatgggagtgatag \\
\hline EgSEP1 & gaagcttaaagctcgttatgaagmcctcc & ccgtgtagatctgatttgcttcaaagaca \\
\hline EgSEP2 & ctcgttatgaagccetacaacggtcac & atctgatttgcttcagcgacgtatcg \\
\hline EgSEP3 & aagcccgatatgaagctctacaacgg & ccgcgtagaacggatttgcttaagtgac \\
\hline EgSEP4 & aagcccgctatgaagctctacaaagg & ccgggtagaacggatttgcttaagcgac \\
\hline EgSEP5 & atggtcttctggatggtagtcagcca & gtgataacgttgaaggacctcgactct \\
\hline EgSEP6 & tttctctacccatggaaagctc & tttcaagtgttcctgtcatgct \\
\hline$E g U b i$ & aagtttgcttcaagctttgct & ggaacaacagagatataggttcaca \\
\hline
\end{tabular}


using SuperScript III Reverse Transcriptase (Life Technologies, Gaithersburg, MD, USA) or ReverTra Ace qPCR RT Master Mix with gDNA Remover (TOYOBO, Osaka, Japan). For real-time PCR analysis, SYBR Green Realtime PCR Master Mix-Plus-(TOYOBO) and the Eco Real-Time PCR System (Illumina, San Diego, CA, USA) were used. The real-time PCR program consisted of $95^{\circ} \mathrm{C}$ for $1 \mathrm{~min}$ followed by 45 cycles at $95^{\circ} \mathrm{C}$ for $15 \mathrm{~s}, 60^{\circ} \mathrm{C}$ for $30 \mathrm{~s}$, and $72^{\circ} \mathrm{C}$ for $30 \mathrm{~s}$. The primer list is shown in Table 1. Real-time PCR analysis was conducted in three biological replicates, each of which was calculated as the average of three technical replicates.

\section{Results}

Identification of Eustoma MIKC'-type MADS-box genes

Through analysis of the assembled transcripts from E. grandiflorum flowers, 23 MIKC'-type MADSbox genes were identified (Table 2). Based on protein sequence similarity, they were grouped into 10 clades, including 4 clades related to flowering time (FLC, SVP, $S O C 1$, and $A G L O)$ and 6 clades related to floral organ identity (AP1/FUL, AP3/DEF, PI/GLO, AG/PLE, STK, and $S E P$ ). Full-length protein sequences could not be obtained for EgSOC1a, EgSOC1b, and EgSVP, so they were not used for phylogenetic and motif analyses. Floral homeotic genes included two A class genes (AP1/FUL clade), six B class genes (AP3/DEF and PI/GLO clades), three $\mathrm{C}$ class genes ( $A G / P L E$ clade), one D class gene

Table 2. List of $M I K C^{c}$-type MADS-box genes identified from Eustoma floral ESTs.

\begin{tabular}{|c|c|c|c|}
\hline No. & Gene Name & Protein Length & Clade \\
\hline 1 & EgPLE1 & 227 & AG/PLE \\
\hline 2 & EgPLE2 & 241 & AG/PLE \\
\hline 3 & $E g P L E 3$ & 256 & AG/PLE \\
\hline 4 & EgSTK & 218 & STK \\
\hline 5 & EgAPla & 245 & AP1/FUL \\
\hline 6 & $E g A P 1 b$ & 242 & AP1/FUL \\
\hline 7 & $E g D E F 1$ & 226 & AP3/DEF \\
\hline 8 & $E g D E F 2$ & 226 & AP3/DEF \\
\hline 9 & $E g D E F 3$ & 230 & $\mathrm{AP} 3 / \mathrm{DEF}$ \\
\hline 10 & EgTM6 & 222 & $\mathrm{AP} 3 / \mathrm{DEF}$ \\
\hline 11 & EgGLOI & 212 & PI/GLO \\
\hline 12 & EgGLO2 & 212 & PI/GLO \\
\hline 13 & EgSEP1 & 237 & SEP \\
\hline 14 & EgSEP2 & 238 & SEP \\
\hline 15 & EgSEP3 & 244 & SEP \\
\hline 16 & EgSEP4 & 244 & SEP \\
\hline 17 & EgSEP5 & 247 & SEP \\
\hline 18 & EgSEP6 & 187 & SEP \\
\hline 19 & EgAGL6 & 254 & AGL6 \\
\hline 20 & $E g F L C$ & 202 & FLC \\
\hline 21 & $E g S V P$ & - & SVP \\
\hline 22 & EgSOCla & - & SOC1 \\
\hline 23 & EgSOC1b & - & SOC1 \\
\hline
\end{tabular}

—; unknown length.
( $S T K$ clade), and six E class genes (SEP clade) (Fig. 1A).

The two $A P 1 / F U L$ clade genes, EgAPla and EgAP1b, showed $62 \%$ amino acid sequence identity and were close to AtAPl and AtFUL, respectively. Arabidopsis and Antirrhinum are known to have two different B class genes, which represent two sub-clades, the AP3/ $D E F$ and $P I / G L O$ clades. Of the six B class genes identified, four belonged to the $A P 3 / D E F$ clade and two belonged to the $P I / G L O$ clade (Fig. 1A). The $A P 3 / D E F$ clade could be further divided into the euAP3 and TM6 sub-clades, although TM6-like genes were absent from Arabidopsis and Antirrhinum (Kramer et al., 1998). In E. grandiflorum, EgDEF1, 2, and 3 were included in the euAP3 sub-clade, while EgTM6 was included in the TM6 sub-clade (Fig. 1A). Amino acid sequences of EgDEF1 and EgDEF2 were 91\% identical and were the same length, but were only $63 \%$ and $64 \%$ identical to EgDEF3, respectively. The amino acid sequence of the two PI/GLO clade genes ( $E g G L O 1$ and $E g G L O 2)$ was almost identical ( $92 \%$ identity).

Three AG/PLE clade genes (EgPLE1, EgPLE2, and $E g P L E 3)$ and one $S T K$ clade gene were identified. The $A G / P L E$ clade can be further divided into the eu $A G$ and PLE sub-clades (Kramer et al., 2004). The three EgPLE genes belonged to the PLE sub-clade (Fig. 1B). EgPLE1 and EgPLE2 had 89\% identical amino acid sequences, while EgPLE3 showed lower similarity to EgPLE1 and 2 (66\% and $71 \%$ identical, respectively). EgPLE3 protein was closely related to the gentian MADS-box gene, GtMADS3 (77\% identity). EgSTK was the only candidate gene for the D class gene and its sequence was the same as EgMADS1 reported by Tzeng et al. (2002).

The six SEP genes (Fig. 1A) were grouped into three pairs (EgSEP1 and EgSEP2, EgSEP3 and EgSEP4, and EgSEP5 and EgSEP6). Each gene of the three pairs showed close sequence similarity $(96 \%$ identical for EgSEP1-2 and EgSEP3-4, and 77\% identical for the aligned region of EgSEP5-6). The two pairs (EgSEP14) belonged to the $S E P 3$ sub-clade, while the other pair (EgSEP5 and EgSEP6) belonged to the SEP4 sub-clade (Fig. 1C).

\section{Clade-specific motifs conserved in Eustoma MADS-box proteins}

MEME motif search identified 16 conserved motifs from E. grandiflorum MADS-box proteins (Fig. 2). Of the four domains in the MIKC ${ }^{c}$-type MADS-box protein, $\mathrm{M}$ (MADS-box) and K (K-box) domains were generally highly conserved, while the other two domains were variable among different MADS-box genes across plant species (Kaufmann et al., 2005). M and $\mathrm{K}$ domains were also conserved in E. grandiflorum MIKC ${ }^{\mathrm{c}}$ MADS-box genes. The MADS-box domain corresponded to motif 1 and part of motif 3 , whereas $\mathrm{K}$ domain corresponded to motif $2,5,7,11,12$, and 15 . Motif 2 was found in all the MADS-box proteins, except for EgFLC (Fig. 2). Motif 4 coincided with the I domain. Motif 13 preceded the 
A

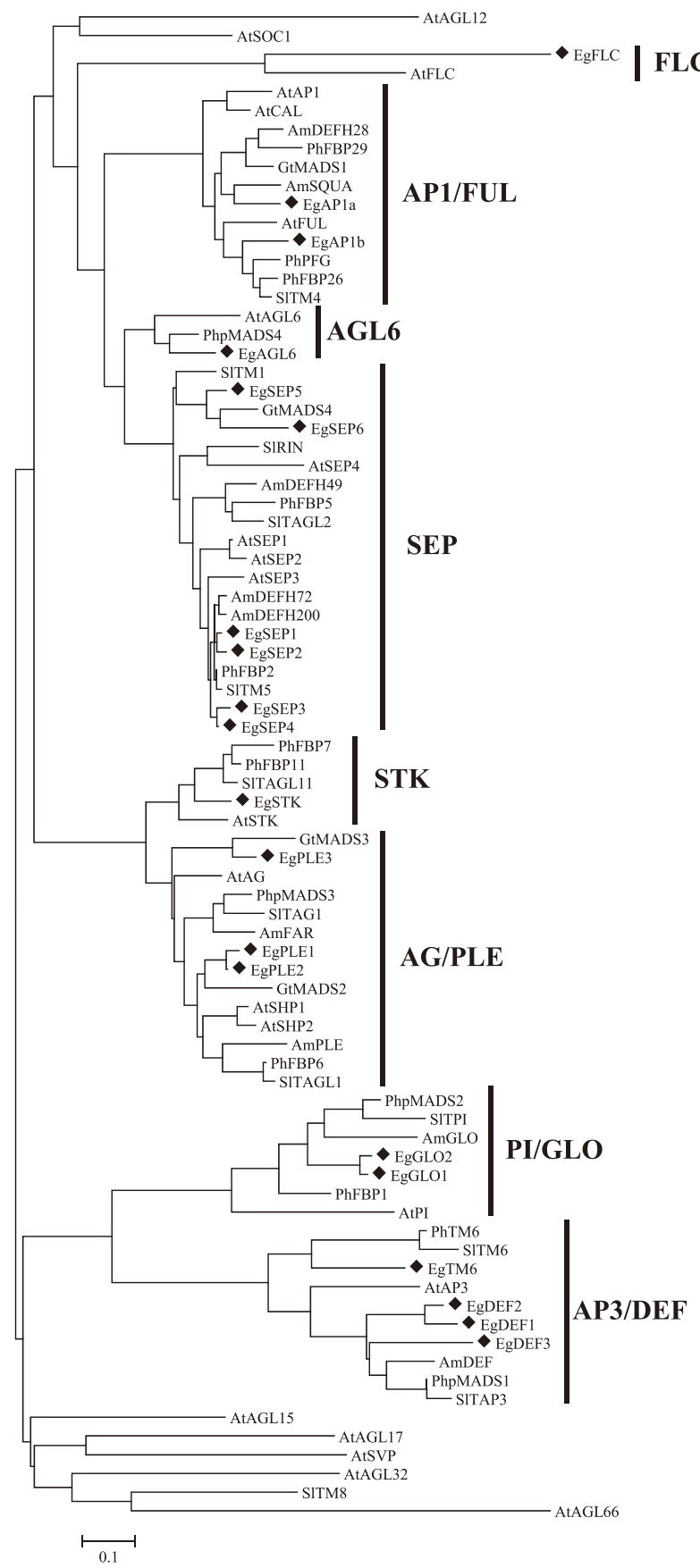

B

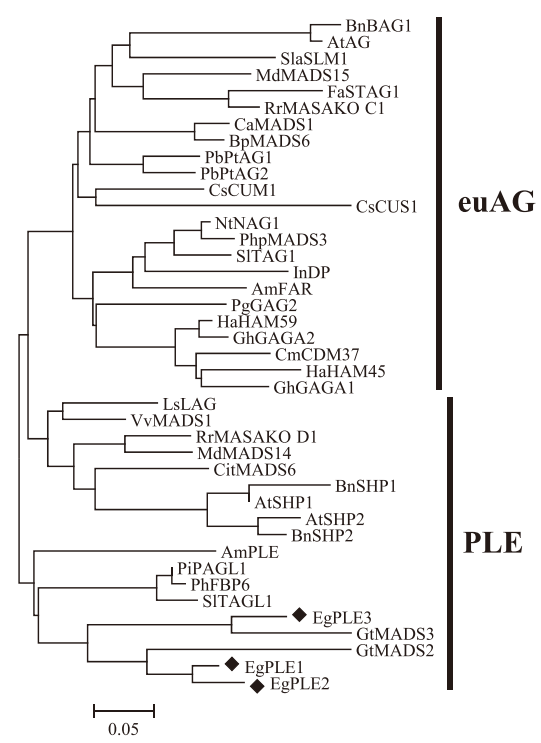

$\mathrm{C}$
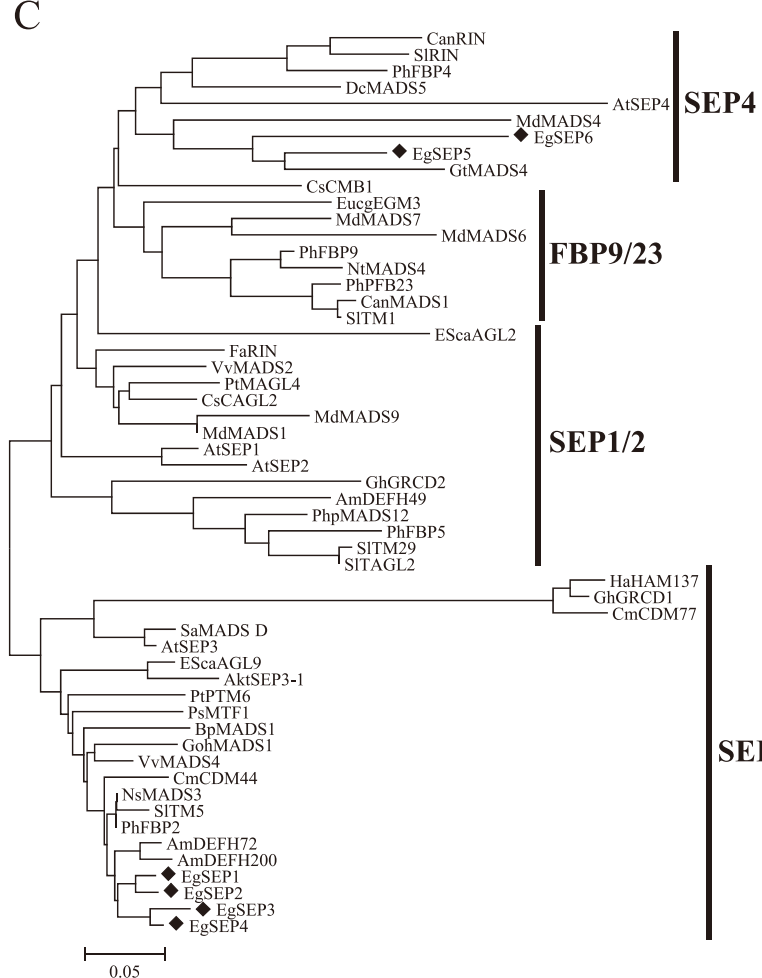

Fig. 1. Phylogenetic trees of MADS-box proteins constructed by neighbor-joining method. A: MIKC-type MADS-box proteins; B: AG/PLE clade proteins; C: SEP clade proteins. $\diamond$ : Erandiflorum genes. Akt, Akebia trifoliata; Am, Antirrhinum majus; At, Arabidopsis thaliana; Bn, Brassica napus; Bp, Betula pendula; Ca, Corylus avellana; Can, Capsicum annuum; Cit, Citrus unshiu; Cm, Chrysanthemum $\times$ morifolium; Cs, Cucumis sativus; Dc, Daucus carota; Eg, Eustoma grandiflorum; Esca, Eschscholzia californica; Eucg, Eucalyptus grandis; Fa, Fragaria $\times$ ananassa; Gh, Gerbera hybrida; Goh, Gossypium hirsutum; Gt, Gentiana triflora; Ha, Helianthus annuus; In, Ipomoea nil; Ls, Liquidambar styraciflua; Md, Malus $\times$ domestica; Ns, Nicotiana sylvestris; Nt, Nicotiana tabacum; Pi, Petunia integrifolia subsp. inflata; Pb, Populus balsamifera subsp. trichocarpa; Pg, Panax ginseng; Ph, Petunia hybrida; Ps, Pisum sativum; Pt, Populus tremuloides; Rr, Rosa rugosa; Sa, Sinapis alba; Sl; Solanum lycopersicum; Sla, Silene latifolia and Vv, Vitis vinifera.

MADS domain, which is specific to AG/PLE-like proteins, such as AG, SHP1 (AGL1), and SHP2 (AGL5) in Arabidopsis (Ma et al., 1991). The other motifs in the C-terminal (motifs 6, 8, 9, 10,14, and 16) were included in the $\mathrm{C}$ domain. Except for motif 10, these motifs corre- sponded to clade-specific motifs. Motif 6 and 14 included SEP I and II motifs, respectively (Zahn et al., 2005). Motif 8 partially matched PI and PI-derived motifs, and motif 16 was part of the euAP3 motif (Kramer et al., 1998). Motif 9 included AG motif I and II (Kramer et al., 
2004). In addition to the predicted motifs by MEME, EgAP1a and EgAP1b proteins contained euAP1 and paleoAP1 motifs (Shan et al., 2007), respectively.

Floral organ-specific expression of ABCDE class genes The ABCDE model predicted that MADS-box genes belonging to the six clades (AP1/FUL, AP3/DEF, PI/ $G L O, A G / P L E, S T K$, and $S E P$ ) are essential for the proper development of floral organs (Krizek and Fletcher, 2005). To see whether the above genes in E. grandiflorum also follow the ABCDE model, their expression patterns in floral organs were evaluated by quantitative real-time

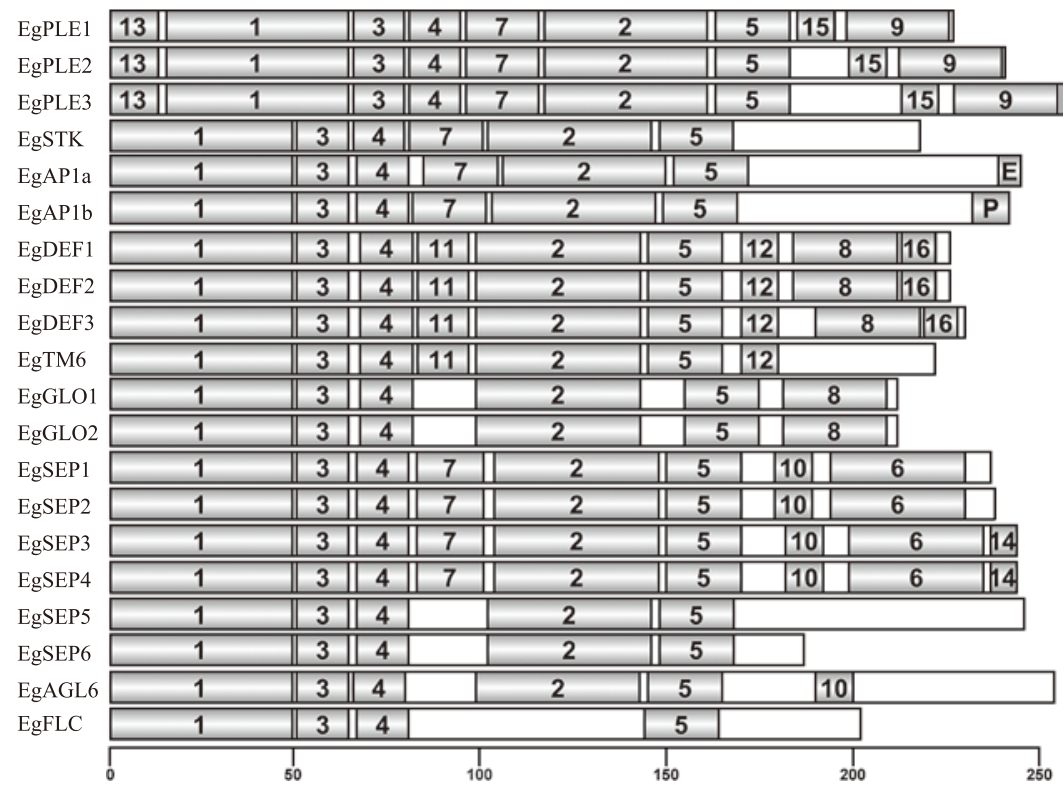

\author{
Motifs in MIKC domains \\ M domain: 1,3 \\ I domain: 4 \\ K domain: 2, 5, 7, 11, 12, 15 \\ C domain : $6,8,9,10,14,16$
}

Other motif: 13

Known clade-specific motifs

SEP I motif: 6

SEP II motif: 14

PI or PI-derived motifs: 8

EuAP3 motif: 16

AG I and II motifs: 9

euAP1 motif: E

paleoAP1 motif: P

Fig. 2. Identification of conserved motifs in E. grandiflorum MADS-box proteins. The motifs were numbered by MEME outputs.
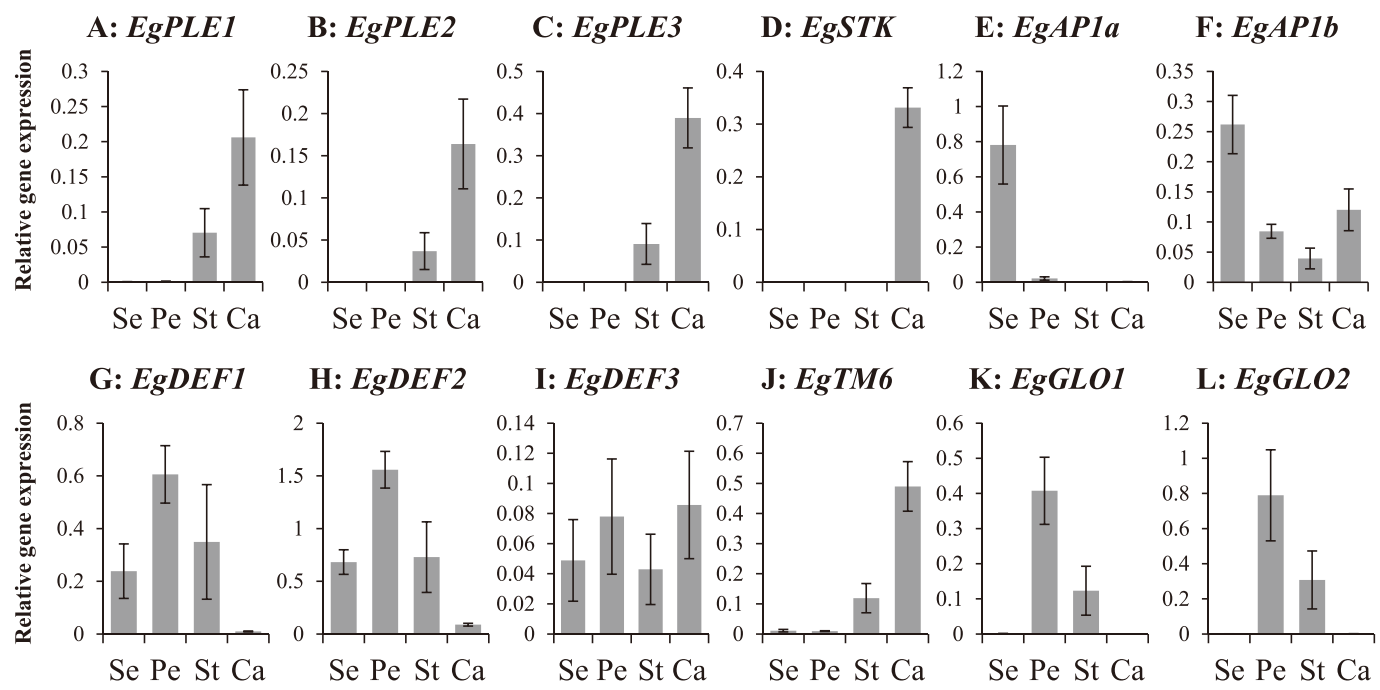

H: $E g D E F 2$

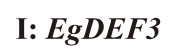

J: EgTM6

K: EgGLO1

$\mathrm{L}: \mathrm{EgGLO} 2$
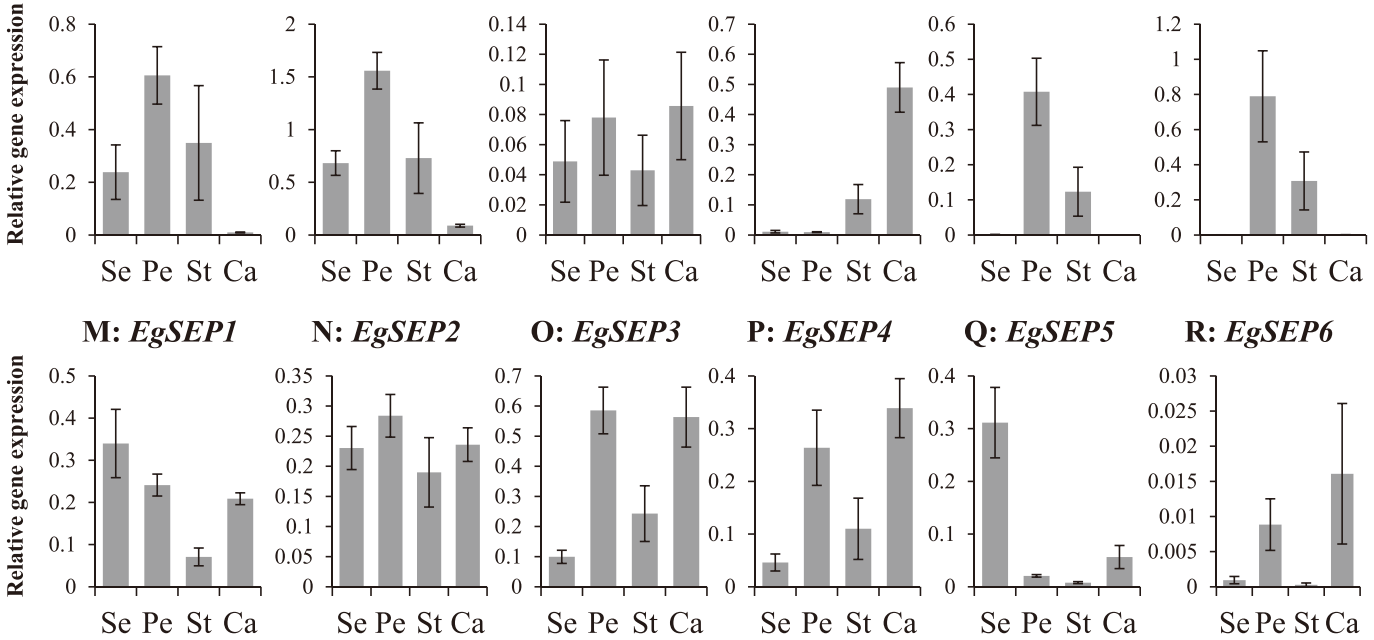

P: EgSEP4

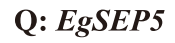

R: EgSEP6

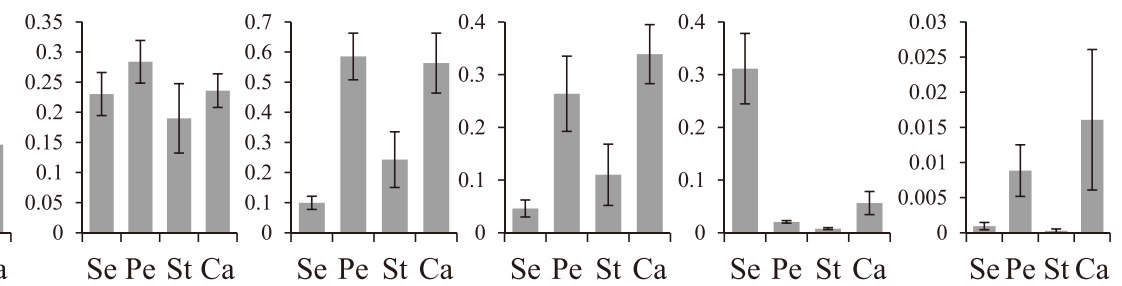

Fig. 3. Quantitative RT-PCR of E. grandiflorum MADS-box genes. EgPLE1 (A), EgPLE2 (B), EgPLE3 (C), EgSTK (D), EgAP1a (E), EgAP1b $(\mathrm{F}), \operatorname{EgDEF} 1(\mathrm{G}), \operatorname{EgDEF} 2(\mathrm{H}), \operatorname{EgDEF} 3(\mathrm{I}), \operatorname{EgTM6}(\mathrm{J}), \operatorname{Eg} G L O 1(\mathrm{~K}), \operatorname{Eg} G L O 2(\mathrm{~L}), \operatorname{EgSEP} 1(\mathrm{M}), \operatorname{EgSEP} 2(\mathrm{~N}), \operatorname{EgSEP} 3(\mathrm{O}), \operatorname{EgSEP} 4$ (P), $\operatorname{EgSEP} 5$ (Q), EgSEP6 (R). Se: sepal, Pe: petal, St: stamen, Ca: carpel. Expression levels were calculated by relative values to ubiquitin $(E g U b i)$. Each value is the mean $\pm \mathrm{SE}(\mathrm{n}=3)$. 
RT-PCR (Fig. 3). All the $A G / P L E$ clade genes were expressed in both stamens and carpels, in agreement with the C-function (Fig. 3A-C). EgSTK was expressed in the carpels (Fig. 3D). In the $A P 1 / F U L$ clade, EgAPla was mainly expressed in the sepals, while $E g A P 1 b$ was also expressed in three other whorls (Fig. 3E, F). Of the three euAP3 sub-clade genes, EgDEF1 and EgDEF2 exhibited similar expression patterns, predominantly in the petals, followed by the sepals and stamens, and faintly in the carpels (Fig. $3 \mathrm{G}, \mathrm{H}$ ). EgDEF3 expression was also detected in all four whorls (Fig. 3I). EgTM6, the paleo $A P 3$ gene, was expressed strongly in the carpels and secondly in the stamens (Fig. 3J). Both PI/GLO clade genes, EgGLO1 and EgGLO2, were expressed in the petals and stamens (Fig. 3K, L). Meanwhile, EgSEP genes showed various expression patterns. Both EgSEP1 and $E g S E P 2$ were expressed in all four whorls, but their expression patterns were different. EgSEP1 expression was the highest in the sepals, whereas EgSEP2 expression was not different among the four whorls (Fig. 3M, $\mathrm{N})$. On the other hand, EgSEP3 and EgSEP4 exhibited similar expression patterns, relatively higher in the petals and carpels (Fig. 3O, P). EgSEP5 was expressed predominantly in the sepals (Fig. 3Q). This expression pattern was quite different from that of EgSEP6, which was expressed strongly in the petals and carpels (Fig. 3R).

\section{Discussion}

Identification of MIKC $C^{c}$-type MADS-box genes in E. grandiflorum

Recent advances in whole-genome sequencing have enabled genome-wide analyses of the MADS-box gene family in economically important crops, such as in grapevine (Diaz-Riquelme et al., 2009), maize, sorghum (Zhao et al., 2011), cucumber (Hu and Liu, 2012), and soybean (Shu et al., 2013). The Arabidopsis, grapevine, and cucumber genomes contained 39, 38, and $30 \mathrm{MIKC}$ type MADS-box genes, respectively (Diaz-Riquelme et al., 2009; Hu and Liu, 2012; Pařenicová et al., 2003). In this study, we identified $23 \mathrm{MIKC}^{\mathrm{c}}$-type MADSbox genes expressed in the Eustoma flower (Table 2). Phylogenetic analysis suggested that 18 of these genes were orthologues of floral homeotic genes (Fig. 1A).

\section{AP1/FUL clade genes}

The two A class genes, EgAPla and EgAP1b, were grouped into the $A P 1 / F U L$ clade. Litt and Irish (2003) reported that $A P 1 / F U L$ clade genes could be divided into euAP1, euFUL and FUL-like sub-clades. While many of the euAPl genes were expressed in specific organs, euFUL and $F U L$-like genes were found to be expressed in various organs in many species (Shan et al., 2007). In $E$. grandiflorum, the eu $A P 1$ sub-clade $E g A P 1 a$ exhibited sepal-specific expression, while the euFUL/FUL-like sub-clade $E g A P 1 b$ exhibited broad expression patterns (Fig. 3E, F). In addition, EgAP1a protein contained
euAP1 motif, which was reported to be specific to the euAP1 sub-clade, whereas EgAP1b contained paleoAP1 motif, which was shared in the euFUL sub-clade and SEP genes (Litt and Irish, 2003; Shan et al., 2007). The expression pattern and motif conservation supported the diversification of EgAP1 genes into euAP1 and euFUL/ FUL-like sub-clades.

\section{AP3/DEF and PI/GLO clade genes}

According to the ABC model, B class genes, corresponding to $A P 3 / D E F$ and $P I / G L O$ clades, function in petal and stamen identities (Krizek and Fletcher, 2005). In E. grandiflorum, two PI/GLO clade genes, EgGLOI and $E g G L O 2$, were expressed in the petals and stamens, as expected by their putative B-function (Fig. 3K, L). However, the expressions of the three $A P 3 / D E F$ genes ( $E g D E F 1,2$, and 3) were not limited to the petals and stamens. $E g D E F 1$ and 2 were expressed mainly in the petals and slightly in the stamens, but they were also expressed in the sepals, and $E g D E F 3$ was expressed in all four whorls (Fig. 3G-I). Therefore, the expression patterns of E. grandiflorum euAP3 genes were not in agreement with the similar B-function of Arabidopsis. Among the three $E g D E F$ genes, $E g D E F 3$ may not have a significant function in the determination of floral organ identity, since the expression level of $E g D E F 3$ was relatively low as compared with other $E g D E F$ s. The expression level often correlates with the function. For instance, loss-of-function analysis of two redundant $\mathrm{C}$ class genes of Petunia hybrida indicated that one $\mathrm{C}$ class gene FBP6 uniquely contributes to style and stigma development, while the other gene PMADS3 exhibited weak expression and did not strongly influence floral organ identity in these organs (Heijmans et al., 2012; Kater et al., 1998).

Another explanation is that the expression patterns of the euAP3 genes ( $E g D E F \mathrm{~s})$ do not influence their functions. In Arabidopsis, AP3 is post-transcriptionally regulated (Jack et al., 1994), and the AP3 function needs PI as an interacting partner (McGonigle et al., 1996). If either EgGLO1 or EgGLO2 protein is necessary for EgDEF functions in E. grandiflorum, the functions of $B$ class genes will be limited to the petals and stamens where EgGLOs are functional.

\section{$A G / P L E$ and $S T K$ clade genes}

Genes of the $A G / P L E$ clade, which comprise C class genes, were reported to be expressed in reproductive organs with a few exceptions (Zahn et al., 2006). This clade included three EgPLE genes (Fig. 1B) that not only shared similar DNA and protein sequences, but also similar expression patterns specific to the stamens and carpels (Fig. 3A-C). These results suggest that EgPLE1-3 genes function as $\mathrm{C}$-class genes redundantly.

$A G / P L E$ and STK (AGL11) clades were considered to have an identical origin, but diverged into two subclades and were neo-functionalized after gene duplica- 
tion (Kramer et al., 2004; Zahn et al., 2006). Further gene duplication in the $A G / P L E$ clade resulted in the eu $A G$ and PLE sub-clades. In Arabidopsis and Antirrhinum, C-functions were attributed to AtAG and AmPLE, respectively (Bradley et al., 1993; Yanofsky et al., 1990). Although it is also known that Arabidopsis has two PLE-like genes, SHATTERPROOF1 and 2 (SHPI and 2), and Antirrhinum has an euAG-like gene, FARINELLI $(F A R)$, these genes do not mainly contribute to their C-functions (Davies et al., 1999; Liljegren et al., 2000). In contrast, in Petunia hybrida and Nicotiana benthamiana, both eu $A G$ and PLE sub-clade genes seem to retain C-functions (Fourquin and Ferrándiz, 2012; Heijmans et al., 2012). In E. grandiflorum, all of the putative C-class genes were grouped into the PLE subclade and no eu $A G$ sub-clade genes could be identified, suggesting that only PLE sub-clade genes specify the C-function.

EgSTK, a putative D class gene, was expressed in the carpels (Fig. 3D). The expression pattern was the same as in Arabidopsis, where the STK expression was limited to the ovules (Pinyopich et al., 2003; Tzeng et al., 2002). The results suggested that EgSTK can specify the ovule identity.

\section{SEP clade genes}

In addition to the $\mathrm{ABCD}$ class genes, $\mathrm{E}$ class genes are essential for specifying floral organ identities by forming heterodimers as predicted by the quartet model. Arabidopsis plants have four redundant SEP genes, since single, double, and triple mutants of SEP genes do not show any or full defects in the floral phenotype, but the quadruple sep1sep2sep3sep 4 mutant develops leaf-like organs (Ditta et al., 2004; Pelaz et al., 2000). However, functional diversification of SEP-like genes was reported in other plants (Malcomber and Kellogg, 2005). In tomato, the SEP-like gene, RIN, is necessary for fruit ripening, although it might be also involved in floral organ development (Ito et al., 2008). In Gerbera hybrida, two SEP homologues, GRCD1 and GRCD2, were predicted to specify stamen and carpel identities, respectively, while GRCD4 and GRCD5 were proposed to act as general E-function regulators displaying hublike positions in the interaction network of MADSbox proteins (Ruokolainen et al., 2010). These reports suggest that some SEP genes are functionally specialized to specific whorls, while others conserve the general E-functions. In E. grandiflorum, the transcripts of EgSEP1-4 were detected in all four whorls, indicating their functions are not specific to certain floral organs (Fig. 3M-P). By contrast, EgSEP5 was preferentially expressed in the sepals and EgSEP6 in the petals and carpels (Fig. 3Q, R), suggesting that these SEP4-like genes have specialized functions in specific floral organs.

\section{Gene duplication in BCE class genes}

The basic mechanism of floral organ specification and patterning regulated by MADS-box genes was found to be conserved in angiosperms (Krizek and Fletcher, 2005). This suggested that diversified flower forms might have evolved from ancient flowering plants along with the diversification of MADS-box genes. Evolutional changes in MADS-box genes include gene duplication and neo-functionalization of the duplicated genes, as well as the changes in protein-protein interactions that may contribute to the formation of MADS-box protein heterodimers (Immink et al., 2010).

From E. grandiflorum, as many as 18 MADS-box genes for $\mathrm{ABCDE}$ class gene clades were identified, whereas some of the orthologues for Arabidopsis floral MADS-box genes could not be identified. The phylogenetic tree suggested the presence of three $A P 3$ orthologues, two $P I$ orthologues and four SEP3 orthologues, while no eu $A G$ clade genes (Fig. 1).

Among the 23 identified MADS-box genes identified, multiple genes showed close sequence similarity. $S E P$ genes, in particular, could be grouped into three pairs (Fig. 1C). EgSEP3 and 4 genes shared the same protein lengths, presence of specific motifs and similar expression patterns (Table 2; Figs. 2 and 3). In addition, EgPLE1-2, EgDEF1-2, and EgGLO1-2 showed very close sequence similarities and similar expression patterns. The presence of closely related MADS-box genes indicates the recent occurrence of gene duplication events. Duplicated genes would redundantly function to prevent unusual specification of floral organ identity under the loss of one of the duplicated genes, but the subsequent diversification of these genes would contribute to the evolution of floral architecture, as suggested for SEP clade genes by Malcomber and Kellogg (2005).

\section{Conclusion}

Our results showed the conservation and diversification of MIKC ${ }^{\mathrm{c}}$-type MADS-box genes during evolution in E. grandiflorum. We identified $23 \mathrm{MIKC}^{\mathrm{c}}$-type MADS-box genes from Eustoma floral transcriptome. These genes were classified as floral homeotic genes, including the $\mathrm{ABCDE}$ genes, and flowering time genes. Phylogenetic analysis suggested that B, C, and E class genes have experienced recent gene duplication events. The identified genes contained some clade-specific motifs. The expression patterns of PI/GLO and PLE clade genes were conserved as predicted by the $\mathrm{ABC}$ model. However, diversified expressions were observed in $A P 3 / D E F$ and $S E P$ clade genes. Transcripts of some genes were observed not only in the whorls predicted from the ABCDE model, but also in other whorls. Such discordance suggested the functional diversification of these genes, but may also result from the flower developmental stages at which the gene expression was analyzed in this study. At later developmental stages, the expression domains of floral homeotic genes can sometimes extend more broadly. In Arabidopsis, for example, AtSEP3 is not expressed in sepal primordia, but is 
on the adaxial side of sepals at later stages (Mandel and Yanofsky, 1998). AtAP3 were also expressed in mature ovules (Jack et al., 1992). In some species, euAPl-like genes were also expressed in the carpels (Shan et al., 2007). To confirm the significant function of the MADSbox genes identified in this study, it is necessary to determine their spatial and temporal expression patterns in floral organ primordia.

\section{Literature Cited}

Alvarez-Buylla, E. R., S. Pelaz, S. J. Liljegren, S. E. Gold, C. Burgeff, G. S. Ditta, L. R. de Pouplana, L. Martínez-Castilla and M. F. Yanofsky. 2000. An ancestral MADS-box gene duplication occurred before the divergence of plants and animals. Proc. Natl. Acad. Sci. USA 97: 5328-5333.

Bailey, T. L., N. Williams, C. Misleh and W. W. Li. 2006. MEME: discovering and analyzing DNA and protein sequence motifs. Nucl. Acids Res. 34 (Suppl. 2): W369-W373.

Bradley, D., R. Carpenter, H. Sommer, N. Hartley and E. Coen. 1993. Complementary floral homeotic phenotypes result from opposite orientations of a transposon at the plena locus of Antirrhinum. Cell 72: 85-95.

Coen, E. S. and E. M. Meyerowitz. 1991. The war of the whorls: genetic interactions controlling flower development. Nature 353: 31-37.

Davies, B., P. Motte, E. Keck, H. Saedler, H. Sommer and Z. Schwarz-Sommer. 1999. PLENA and FARINELLI: redundancy and regulatory interactions between two Antirrhinum MADS-box factors controlling flower development. EMBO J. 18: 4023-4034.

Ditta, G., A. Pinyopich, P. Robles, S. Pelaz and M. F. Yanofsky. 2004. The SEP4 gene of Arabidopsis thaliana functions in floral organ and meristem identity. Curr. Biol. 14: 1935-1940.

Díaz-Riquelme, J., D. Lijavetzky, J. M. Martínez-Zapater and M. J. Carmona. 2009. Genome-wide analysis of MIKC ${ }^{\mathrm{C}}$-type MADS box genes in grapevine. Plant Physiol. 149: 354-369.

Eddy, S. R. 2009. A new generation of homology search tools based on probabilistic inference. Genome Inform. 23: 205-211.

Fourquin, C. and C. Ferrándiz. 2012. Functional analyses of AGAMOUS family members in Nicotiana benthamiana clarify the evolution of early and late roles of C-function genes in eudicots. Plant J. 71: 990-1001.

Goto, K. and E. M. Meyerowitz. 1994. Function and regulation of the Arabidopsis floral homeotic gene PISTILLATA. Genes Dev. 8: 1548-1560.

Gouzy, J., S. Carrere and T. Schiex. 2009. FrameDP: sensitive peptide detection on noisy matured sequences. Bioinformatics 25: 670-671.

Heijmans, K., K. Ament, A. S. Rijpkema, J. Zethof, M. WoltersArts, T. Gerats and M. Vandenbussche. 2012. Redefining C and D in the petunia ABC. Plant Cell 24: 2305-2317.

$\mathrm{Hu}, \mathrm{L}$. and S. Liu. 2012. Genome-wide analysis of the MADS-box gene family in cucumber. Genome 55: 245-256.

Immink, R. G. H., K. Kaufmann and G. C. Angenent. 2010. The 'ABC' of MADS domain protein behaviour and interactions. Semin. Cell Dev. Biol. 21: 87-93.

Ito, Y., M. Kitagawa, N. Ihashi, K. Yabe, J. Kimbara, J. Yasuda, H. Ito, T. Inakuma, S. Hiroi and T. Kasumi. 2008. DNA-binding specificity, transcriptional activation potential, and the rin mutation effect for the tomato fruit-ripening regulator RIN. Plant J. 55: 212-223.

Jack, T., L. L. Brockman and E. M. Meyerowitz. 1992. The homeotic gene APETALA3 of Arabidopsis thaliana encodes a MADS box and is expressed in petals and stamens. Cell 68: 683-697.

Jack, T., G. L. Fox and E. M. Meyerowitz. 1994. Arabidopsis homeotic gene APETALA 3 ectopic expression: Transcriptional and posttranscriptional regulation determine floral organ identity. Cell 76: 703-716.

Jofuku, K. D., B. Den Boer, M. Van Montagu and J. K. Okamuro. 1994. Control of Arabidopsis flower and seed development by the homeotic gene APETALA2. Plant Cell 6: 1211-1225.

Kater, M. M., L. Colombo, J. Franken, M. Busscher, S. Masiero, M. M. Van Lookeren Campagne and G. C. Angenent. 1998. Multiple AGAMOUS homologs from cucumber and petunia differ in their ability to induce reproductive organ fate. Plant Cell 10: 171-182.

Kaufmann, K., R. Melzer and G. Theißen. 2005. MIKC-type MADS-domain proteins: structural modularity, protein interactions and network evolution in land plants. Gene 347: 183-198.

Kawabata, S., Y. Li and K. Miyamoto. 2012. EST sequencing and microarray analysis of the floral transcriptome of Eustoma grandiflorum. Sci. Hortic. 144: 230-235.

Kramer, E. M., R. L. Dorit and V. F. Irish. 1998. Molecular evolution of genes controlling petal and stamen development: duplication and divergence within the APETALA3 and PISTILLATA MADS-box gene lineages. Genetics 149: 765-783.

Kramer, E. M., M. A. Jaramillo and V. S. Di Stilio. 2004. Patterns of gene duplication and functional evolution during the diversification of the AGAMOUS subfamily of MADS box genes in angiosperms. Genetics 166: 1011-1023.

Krizek, B. A. and J. C. Fletcher. 2005. Molecular mechanisms of flower development: an armchair guide. Nat. Rev. Genet. 6: 688-698.

Larkin, M., G. Blackshields, N. Brown, R. Chenna, P. A. McGettigan, H. McWilliam, F. Valentin, I. M. Wallace, A. Wilm and R. Lopez. 2007. Clustal W and Clustal X version 2.0. Bioinformatics 23: 2947-2948.

Liljegren, S. J., G. S. Ditta, Y. Eshed, B. Savidge, J. L. Bowman and M. F. Yanofsky. 2000. SHATTERPROOF MADS-box genes control seed dispersal in Arabidopsis. Nature 404: 766-770.

Litt, A. and V. F. Irish. 2003. Duplication and diversification in the APETALA1/FRUITFULL floral homeotic gene lineage: implications for the evolution of floral development. Genetics 165: 821-833.

Ma, H., M. F. Yanofsky and E. M. Meyerowitz. 1991. AGL1-AGL6, an Arabidopsis gene family with similarity to floral homeotic and transcription factor genes. Genes Dev. 5: 484-495.

Malcomber, S. T. and E. A. Kellogg. 2005. SEPALLATA gene diversification: brave new whorls. Trends Plant Sci. 10: 427-435.

Mandel, M. A., C. Gustafson-Brown, B. Savidge and M. F. Yanofsky. 1992. Molecular characterization of the Arabidopsis floral homeotic gene APETALA1. Nature 360: 273-277.

Mandel, M. A. and M. F. Yanofsky. 1998. The Arabidopsis AGL9 MADS box gene is expressed in young flower primordia. Sex. Plant Reprod. 11: 22-28.

McGonigle, B., K. Bouhidel and V. F. Irish. 1996. Nuclear localization of the Arabidopsis APETALA3 and PISTILLATA homeotic gene products depends on their simultaneous expression. Genes Dev. 10: 1812-1821.

Pařenicová, L., S. de Folter, M. Kieffer, D. S. Horner, C. Favalli, J. Busscher, H. E. Cook, R. M. Ingram, M. M. Kater, B. Davies, G. C. Angenent and L. Colombo. 2003. Molecular and phylogenetic analyses of the complete MADS-box transcription factor family in Arabidopsis: new openings to the MADS world. Plant Cell 15: 1538-1551. 
Pelaz, S., G. S. Ditta, E. Baumann, E. Wisman and M. F. Yanofsky. 2000. B and C floral organ identity functions require SEPALLATA MADS-box genes. Nature 405: 200-203.

Pinyopich, A., G. S. Ditta, B. Savidge, S. J. Liljegren, E. Baumann, E. Wisman and M. F. Yanofsky. 2003. Assessing the redundancy of MADS-box genes during carpel and ovule development. Nature 424: 85-88.

Quevillon, E., V. Silventoinen, S. Pillai, N. Harte, N. Mulder, R. Apweiler and R. Lopez. 2005. InterProScan: protein domains identifier. Nucleic Acids Res. 33: W116-W120.

Ren, J., L. Wen, X. Gao, C. Jin, Y. Xue and X. Yao. 2009. DOG 1.0: illustrator of protein domain structures. Cell Res. 19: 271-273.

Ruokolainen, S., Y. P. Ng, V. A. Albert, P. Elomaa and T. H. Teeri. 2010. Large scale interaction analysis predicts that the Gerbera hybrida floral E function is provided both by general and specialized proteins. BMC Plant Biol. 10: 129.

Shan, H., N. Zhang, C. Liu, G. Xu, J. Zhang, Z. Chen and H. Kong. 2007. Patterns of gene duplication and functional diversification during the evolution of the API/SQUA subfamily of plant MADS-box genes. Mol. Phylogenet. Evol. 44: 26-41.

Shu, Y., D. Yu, D. Wang, D. Guo and C. Guo. 2013. Genome-wide survey and expression analysis of the MADS-box gene family in soybean. Mol. Biol. Rep. 40: 3901-3911.

Sommer, H., J. P. Beltran, P. Huijser, H. Pape, W. E. Lönnig, H. Saedler and Z. Schwarz-Sommer. 1990. Deficiens, a homeotic gene involved in the control of flower morphogenesis in Antirrhinum majus: the protein shows homology to transcription factors. EMBO J. 9: 605-613.

Tamura, K., D. Peterson, N. Peterson, G. Stecher, M. Nei and S.
Kumar. 2011. MEGA5: molecular evolutionary genetics analysis using maximum likelihood, evolutionary distance, and maximum parsimony methods. Mol. Biol. Evol. 28: 27312739.

Tzeng, T. Y., H. Y. Chen and C. H. Yang. 2002. Ectopic expression of carpel-specific MADS box genes from lily and lisianthus causes similar homeotic conversion of sepal and petal in Arabidopsis. Plant Physiol. 130: 1827-1836.

Yanofsky, M. F., H. Ma, J. L. Bowman, G. N. Drews, K. A. Feldmann and E. M. Meyerowitz. 1990. The protein encoded by the Arabidopsis homeotic gene agamous resembles transcription factors. Nature 346: 35-39.

Zahn, L. M., H. Kong, J. H. Leebens-Mack, S. Kim, P. S. Soltis, L. L. Landherr, D. E. Soltis, C. W. dePamphilis and H. Ma. 2005. The evolution of the SEPALLATA subfamily of MADSBox genes: a preangiosperm origin with multiple duplications throughout angiosperm history. Genetics 169: 2209-2223.

Zahn, L. M., J. H. Leebens-Mack, J. M. Arrington, Y. Hu, L. L. Landherr, C. W. dePamphilis, A. Becker, G. Theissen and H. Ma. 2006. Conservation and divergence in the AGAMOUS subfamily of MADS-box genes: evidence of independent sub- and neofunctionalization events. Evol. Dev. 8: 30-45.

Zhang, Y. and Y. Sun. 2011. HMM-FRAME: accurate protein domain classification for metagenomic sequences containing frameshift errors. BMC bioinformatics 12: 198.

Zhao, Y., X. Li, W. Chen, X. Peng, X. Cheng, S. Zhu and B. Cheng. 2011. Whole-genome survey and characterization of MADSbox gene family in maize and sorghum. Plant Cell Tissue Organ Cult. 105: 159-173. 